\title{
Frenkel Defects and Interstitial Atoms in Periclase (MgO) at High Temperature by Molecular Dynamics Simulation
}

\author{
Yasuhiro UEDA ${ }^{\mathrm{a}}$ and Akira MIYAKE ${ }^{\mathrm{a}^{*}}$ \\ ${ }^{a}$ Graduate School of Science, Kyoto University, Kitashirakawa-Oiwakecho, Kyoto 606-8502, Japan \\ *e-mail:miya@kueps.kyoto-u.ac.jp
}

(Received: April 22, 2015; Accepted for publication: June 12, 2015; Online publication: October 1, 2015)

An analysis program was newly developed to explore the vacancies and interstitial atoms and to determine atomic migration, i.e., atomic diffusion. We applied this program to the results calculated by the molecular dynamics (MD) simulation of periclase (MgO) in which Schottky defects (vacancies) were not initially introduced. Generation, migration and extinction of Frenkel defects and interstitial atoms for only magnesium ion were first observed at high temperature in this MD system and they strongly corresponded to the change of the mean square distance (MSD) of magnesium ion in MD system. On the other hand, we could not observe Frenkel defects and interstitial atoms for oxygen ion and MSD value of oxygen ion had almost constant value. Generation, migration and extinction of Frenkel defects and interstitial atoms cannot be ignored for the diffusion process at high temperature.

Keyword: Periclase (MgO), Molecular dynamics simulation, Frenkel defect, Interstitial atom, Diffusion

\section{Introduction}

Diffusion process is a very important physical property not only in material science but also in earth and planetary sciences and controls many kinetic phenomena such as creep of solid phases and chemical mass transportation. Periclase $(\mathrm{MgO})$ is thought to be one of the important component minerals of the Earth's lower mantle. Therefore, diffusion in periclase has been studied in the material science and earth science. For example, Van Orman et al. [1] measured self-diffusion coefficients of periclase at 15-25 GPa pressures. Moreover, the diffusion coefficient in periclase was also calculated by computer simulation. For example, Vočadlo et al. [2], calculated self-diffusion coefficients by computer simulations based upon lattice dynamics and Ito and Toriumi [3] calculated the pressure effect by molecular dynamics (MD) calculations. Point defects play a very important role in diffusion processes. The important point defect is not only Schottky defects but also Frenkel defects and interstitial atoms. In the previous studies such as Ito and Toriumi [3], however, the only Schottky defects such as vacancies of magnesium and oxygen pair were initially introduced into the
MD structure, but Frenkel defects and interstitial atoms have not been mentioned from the simulation results. Furthermore, the migration of individual atoms and vacancies could not be analyzed in detail.

In the present study, we developed newly an analysis program to explore the vacancies and interstitial atoms and to determine the atomic migration. We first report that Frenkel defects and interstitial atoms generate and become extinct at high temperature even by simulation results from an MD crystal without Schottky defects in initial structure.

\section{Method}

\subsection{Molecular dynamics simulation}

In the present study, an interatomic potential model was employed, where the interatomic potential function $\left(\Phi_{i j}\right)$ between two atoms, $i$-th and $j$-th atoms, consists of the Coulombic, the short range repulsion, the van der Waals attraction, and the Morse potential terms as given by 


$$
\begin{aligned}
& \varphi_{i j}\left(r_{i j}\right)=\frac{z_{i} z_{j} e^{2}}{r_{i j}}+f_{0}\left(b_{i}+b_{j}\right) \exp \left(\frac{a_{i}+a_{j}-r_{i j}}{b_{i}+b_{j}}\right)-\frac{c_{i} c_{j}}{r_{i j}^{6}} \\
& +D_{i j}\left\{\exp \left[-2 \beta_{i j}\left(r_{i j}-r_{i j}^{*}\right)\right]-2 \exp \left[-\beta_{i j}\left(r_{i j}-r_{i j}^{*}\right)\right]\right\},
\end{aligned}
$$

where $r_{i j}$ is an interatomic distance, $f_{0}\left(=6.9511 \times 10^{-11} \mathrm{~N}\right)$ a constant, $e$ the electronic charge, $z, a, b$ and $c$ parameters for each atomic species, and $D_{i j}, \beta_{i j}$ and $r_{i j}^{*}$ parameters for cationanion pairs. The parameters used in the present study were empirically evaluated by Miyake [4]. MD simulations for silicate minerals such as enstatite $\left(\mathrm{MgSiO}_{3}\right)$ and cordierite $\left(\mathrm{Mg}_{2} \mathrm{Al}-\right.$ ${ }_{4} \mathrm{Si}_{5} \mathrm{O}_{18}$ ) were sucessfully carried out using this parameter set in the previous studies (e.g., Miyake et al. [5]; Miyake [6],).

The present MD simulations were carried out by using a program MXDORTO and MXDORTOP (Kawamura [7],), by applying the Ewald method for the summations of Coulombic interactions, and by integrating the atomic motion by means of Verlet's algorithm $(\Delta t=2.0$ femto seconds $)$. Periodic boundary conditions were imposed with the MD basic cell. The MD basic cell was composed of 216 unit cells $\left(a_{\mathrm{MD}}=b_{\mathrm{MD}}=c_{\mathrm{MD}}\right.$ $=6 a_{\text {periclase}}$ ) for periclase (containing 1728 atoms). Every MD simulation was carried out by an NPT ensemble, where $N$ is the number of atoms, $P$ pressure and $T$ temperature in the system. Temperature and pressure were controlled by scaling particle velocities and simulating cell parameters, respectively. Temperature range of $300-3000 \mathrm{~K}$ was investigated, while pressure was kept around $1 \mathrm{~atm}(0 \mathrm{GPa})$ throughout the MD simulations. Various crystallographic and physical properties were obtained by the calculations for at least 2 nano seconds (ns) $(=1,000,000$ steps) after annealing for at least 20 ps.

The structure of periclase determined by the X-ray diffraction method (Wyckoff [8],) was adopted as the initial state. Ito and Toriumi [3] prepared pair vacancies of magnesium and oxygen ions (Schottky defects) in MD system of periclase. In the present study, we simulated the perfect MD crystal with no vacancies (no Schottky defects) as the initial state to reveal the appearance of Frenkel defects and interstitial atoms.

\subsection{Analysis program to explore the vacancy and interstitial atom and to determine the atomic migration}

In MD simulation, we can track the motion of each atom because time variation of all atomic positions in structure can be obtained. At high temperature, an atom oscillates hardly and moves too far from the initial position. Consequently, an atom will migrate from the original position to around the neighbored atom and the generation of vacancy (Frenkel defect) and interstitial atom might be expected. In the previous studies, however, Frenkel defect and interstitial atom only from the time variation of atomic position could not be identified and they had not been mentioned. In the present study, we developed newly an analysis program by introducing the original concept as "room," to explore the vacancy and interstitial atom and to determine the atomic migration. We explain this original program in detail below;

\subsubsection{Original concept, "room"}

"Room" is defined as the enclosed space whose dimensions with the half distance of the first neighbor atom from the atomic position in perfect structure. Here, the first neighbor atom is defined as same element. The "room" is individually decided on each element and it is clearly distinguished between "room" of magnesium ion and "room" of oxygen ion. Figure 1 shows the 2 dimensional schematic illustration in the case of pericalse.

\subsubsection{Determination method of atomic "room" address}

For determination of the "room" address where each atom stayed, "room" defined as above was first applied to each snapshot of MD simulation results at each step. We decided basically the address of a "room" to which an atom stays as the atomic "room" address. The schematic illustration was shown in Figure 2.

Here, if the structure changed significantly and the phase transformed to a new phase, we needed to redefine newly "room". Therefore, we calculated the structure factors of MD simulated structure at each simulated condition using the method proposed by Miyake et al. [9] and identified the phase. In the present study, the phase transition could not be confirmed except for melting over $3100 \mathrm{~K}$.

In addition to these procedures, we corrected the decision method of the atomic "room" address in the following methods, because we distinguish between the temporary migration of an atom to the other "room" due to the atomic thermal vibration at high temperature and the migration for the diffusion process. If the "room" to which the atom $x$ stays at $i-1$ step ("room-A") 
(a)

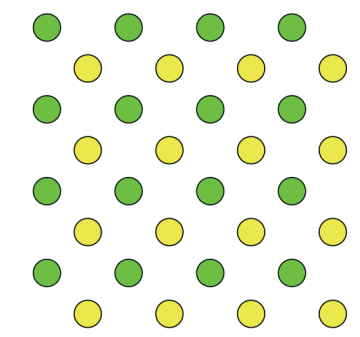

(b)

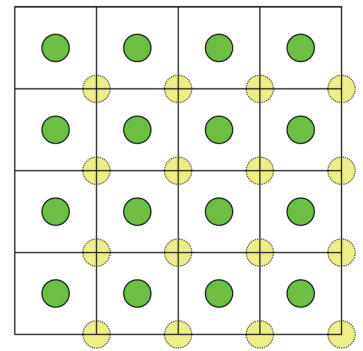

(c)

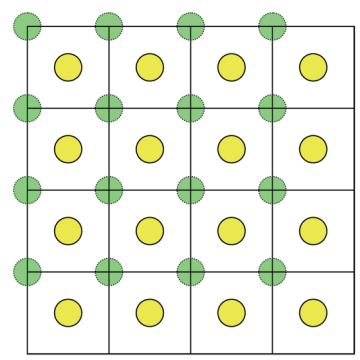

Figure 1. Schematic illustration of (a) MgO crystal, (b) "room" of O ion and (c) "room" of magnesium ion, defined in this study. magnesium ions are yellow and $\mathrm{O}$ ions are green.

(a)

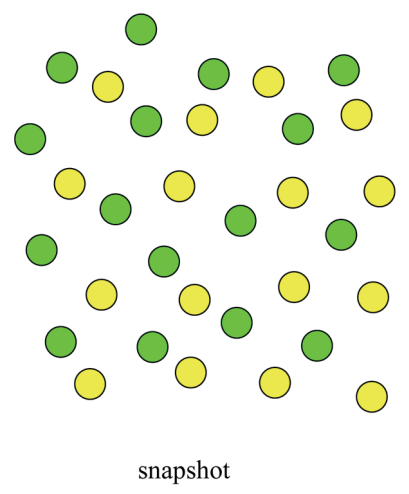

(b)

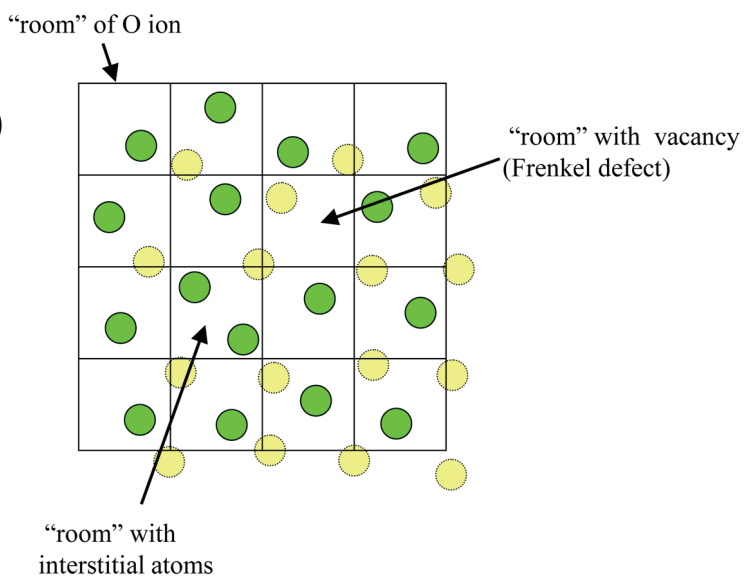

Figure 2. Schematic illustration of (a) one snapshot in MD simulation results and (b) the "room" of O ion. Mg ions are yellow and $\mathrm{O}$ ions are green. The "room" with Frenkel defect has no O ion in the "room." The "room" with two O ions has the interstitial atoms.

was different from the "room" at $i$ step ("room-B"), we applied the correction as following. At arbitrary $j$ step after $i$ step $(i<j)$, if the atom $\mathrm{x}$ did not go back to the "room-A" and the angle $\mathrm{ABX}_{j}$ is larger than 90 degrees $\left(\angle \mathrm{ABX}_{j}>90^{\circ}\right)$, the atom $x$ stays to "room-B" after $i$ step. Here, the center positions for "room-A" and "room-B" were defined as positions A and B, respectively, and the atomic position of atom $x$ was defined as position $\mathrm{X}_{j}$ at $j$ step. $\angle \mathrm{ABX}_{j}>90^{\circ}$ means that atom $x$ moved beyond the half of "room-B" at $j$ step. At $j$ step after $i$ step, on the other hand, if the atom $x$ went back to the "room-A" without $\angle \mathrm{ABX}_{j}>90^{\circ}$, the atom $x$ temporarily migrated to "room$\mathrm{B}$ " and kept to stayed in "room-A" after $i$ step. This correction means that the "room" where atom $x$ stays at $i$ step depends on the atomic position of atom $x$ not only at $i$ step but also after $i$ step. The schematic illustration of this corrected method was shown in Figure 3.

\subsubsection{Criteria for judgment of vacancy (Frenkel defect), interstitial atom and atomic migration}

After we decided the "room" to which each atom stayed at each step according to determination method as above at first, we judged vacancy (Frenkel defect), interstitial atom, and atomic migration according to the following criteria.

Frenkel defect: if "room" where no atom stayed to exited at certain step, the "room" contained the vacancy (Frenkel defect) at the step (Figure 2b).

Interstitial atom: if "room" where two or more atoms stayed to exited at certain step, the "room" had one/some interstitial atoms at the step (Figure 2b).

Atomic migration: if a "room" where a certain atom stayed to at certain step was different from a "room" at previous step, we judged that the atom migrated at the step 

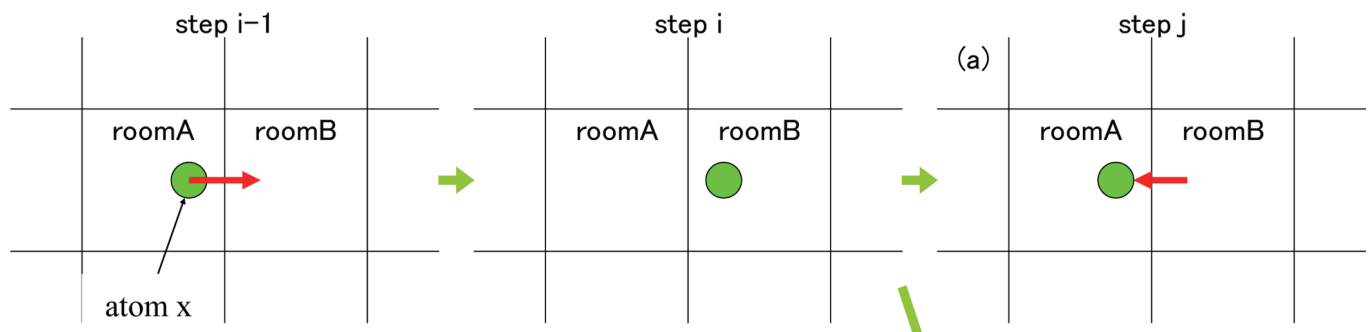

"room" address where atom $x$ belongs at $i$ step; case-(a): "room-A"

case-(b): "room-B"

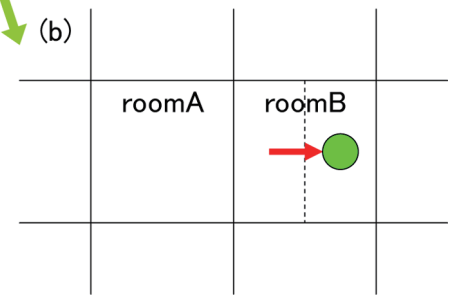

Figure 3. Schematic illustration of the correction method of determination of atomic "room."
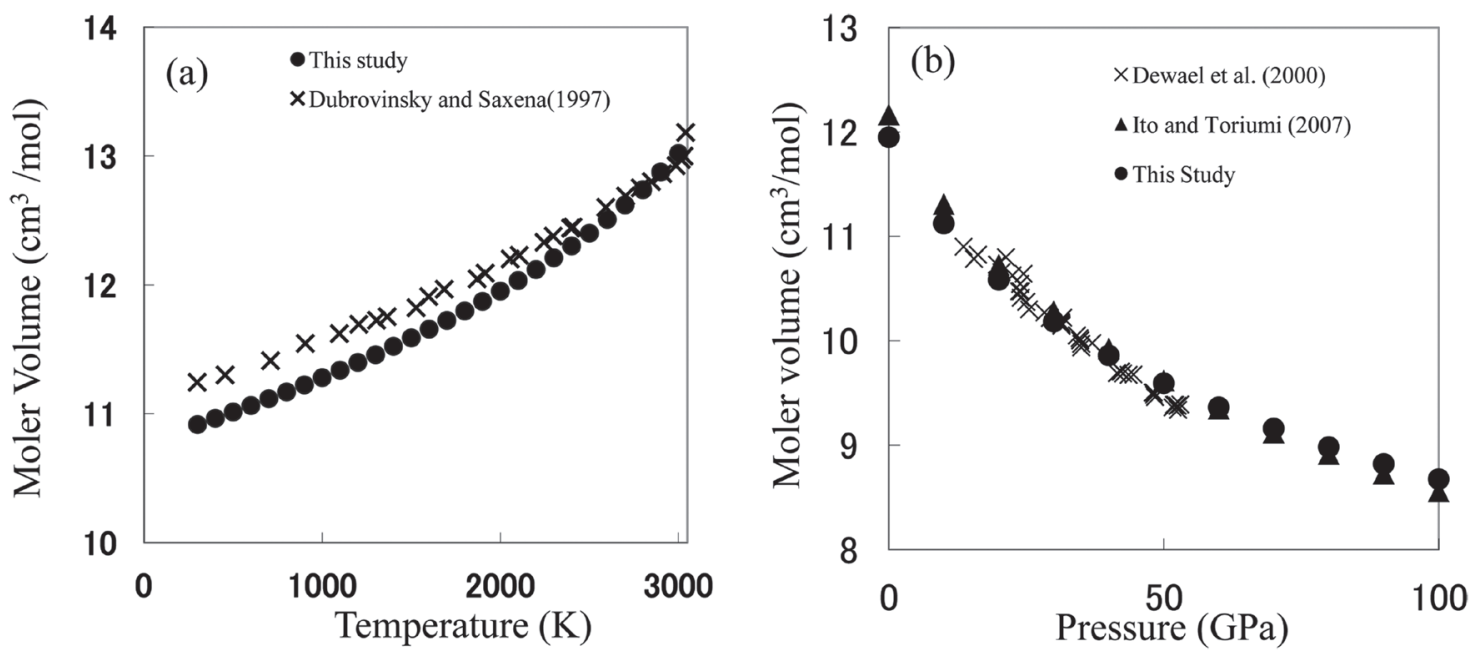

Figure 4. (a) Thermal expansions of molar volumes at $0 \mathrm{GPa}$. (b) Compressions of molar volumes at $2000 \mathrm{~K}$.

\section{Result and discussion}

\subsection{Thermal expansion at $0 \mathrm{GPa}$ and compression at $2000 \mathrm{~K}$}

Figure 4a shows that the thermal expansion derived from the present MD calculation at $0 \mathrm{GPa}$ was compared with the experiments by an X-ray diffraction method (Dubrovinsky and Saxena [10],). Figure $4 \mathrm{~b}$ shows that the present compression under high temperature $(2000 \mathrm{~K})$ was compared with those determined by previous MD calculation (Ito and Toriumi [3],) and by an X-ray diffraction method (Dewael et al. [11]). Both thermal expansion and compression of the molar volumes are in good agreement with those of experiments. Therefore we con- sider that reproduction of the present simulation is good and the self-diffusion process simulated by the present simulation is reliable enough to provide the base data compared with the previous study.

\subsection{Generation, migration and extinction of Frenkel defect and interstitial atom of magnesium ion}

Figure 5 shows a sequence of snapshots of a part of the simulated $\mathrm{MgO}$ structure at $0 \mathrm{GPa}$ and $3000 \mathrm{~K}$. magnesium ions oscillated around the atomic position in lattice (Figure 5a). Then an magnesium ion abruptly migrated to a neighbor position at certain time. As the result, Frenkel defect generated in the former 
(a)

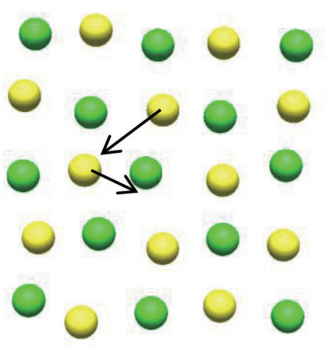

(d)

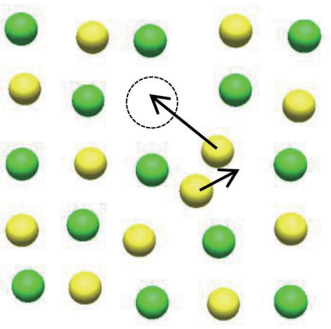

(b)

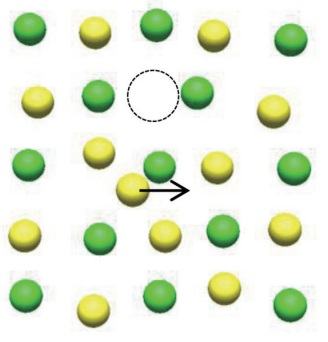

(e)

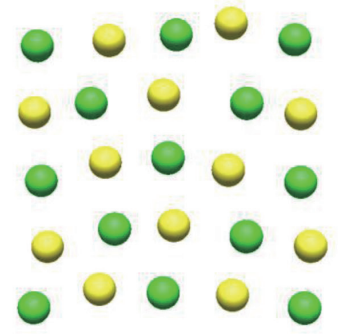

(c)

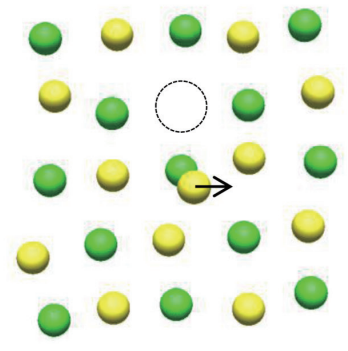

Figure 5. A sequence of snapshots from (a) to (e) of simulated $\mathrm{MgO}$ structure at $0 \mathrm{GPa}$ and $3000 \mathrm{~K} . \mathrm{Mg}$ ions are yellow and $\mathrm{O}$ ions are green. The interval between snapshots is each $80 \mathrm{MD}$ steps. The arrows indicate the motion of the $\mathrm{Mg}$ ion and the dotted circles approximately show the Frenkel defect.

"room" and the interstitial atom formed in the migrated "room" (Figure 5b). After the interstitial atom repeated to migrate to the other neighbor "room" several times (Figure $5 b-5 e$ ), the interstitial atom migrated to "room" with the Frenkel defect and finally both became extinct (Figure 5e).

These results indicated that both the Frenkel defect and interstitial atom generated in the MD structure and that the diffusion process occurred despite the fact that the initial MD cell has no point defects (no vacancy). This phenomenon in MD simulation was the first reported and this proves the usefulness of the analysis program of atomic migration developed in the present study.

\subsection{Comparison between mean square distance (MSD) and appearance frequency of Frenkel defects}

MSD value of ion calculated by MD simulations has the constant value in the case where the ion merely oscillates around the original atomic position in the lattice and the diffusion process does not occur. On the other hand, MSD value increases linearly with time in the case where the diffusion process occurs. MSD in melt increases precipitously and has the distinctly different gradient. If Frenkel defects and interstitial atoms gen- erated, they migrated respectively and the diffusion occurred, that is the MSD values should increase linearly. When the defects and the interstitial atoms met each other, both became extinct and the diffusion process stopped which meant that the MSD became to have constant value. Figure 6 shows the time dependences of MSDs of magnesium and oxygen ions at 0 $\mathrm{GPa}$ and $3000 \mathrm{~K}$ and the durations which the Frenkel defects of magnesium ion were identified in the present simulation. MSD of magnesium ion showed that a repeat of the monotonous increasing and the keeping the constant value and had the stepwise change. Frenkel defects and interstitial ions observed by the present program also showed a repeat of the existences and the extinctions (Figure 6). The monotonous increasing durations of MSD of magnesium ion were in excellent agreement with the duration that Frenkel defects and interstitial atoms generated and migrated. Moreover, the durations with almost constant MSD values also were in agreement with the duration that defects and interstitial atoms could not be observed. These results indicated that the stepwise change of MSD value corresponded to the generation, the migration and the extinction of Frenkel defects and interstitial atoms. In the present study, on the other hand, Frenkel defects and interstitial atoms of oxygen ions did not generate at all simulated conditions and therefore 


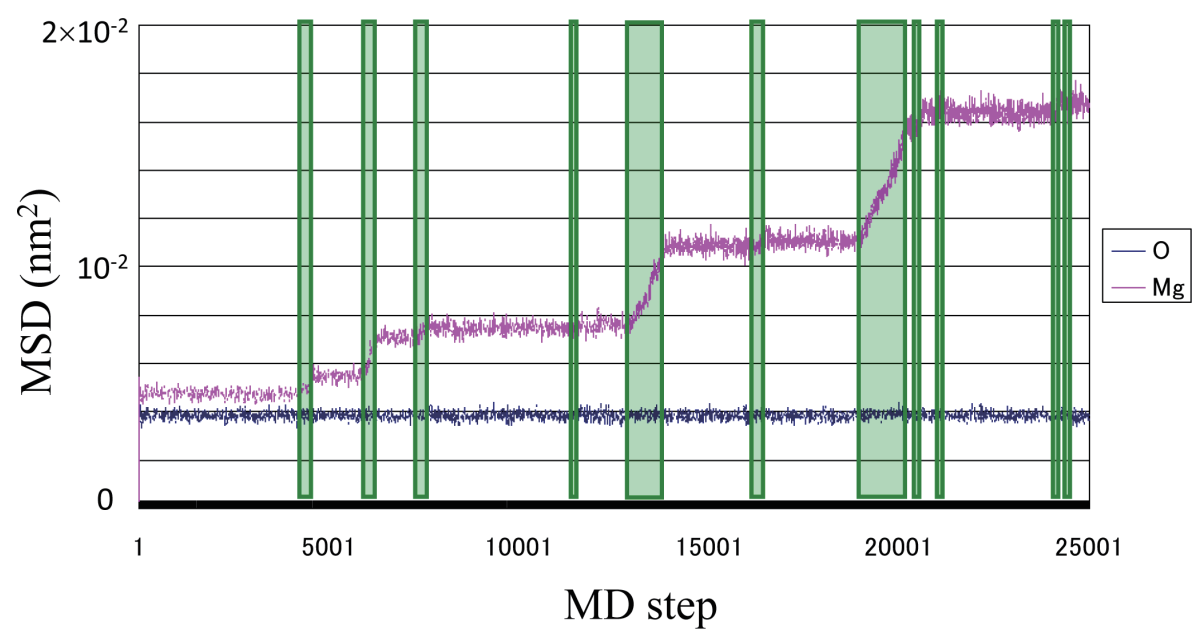

Figure 6. Time dependences of MSD of Mg and $\mathrm{O}$ ions at $0 \mathrm{GPa}$ and $3000 \mathrm{~K}$. Mg ions are yellow and $\mathrm{O}$ ions are green. The indicated areas in green show the MD step ranges of the appearance of Frenkel defect for Mg ion.

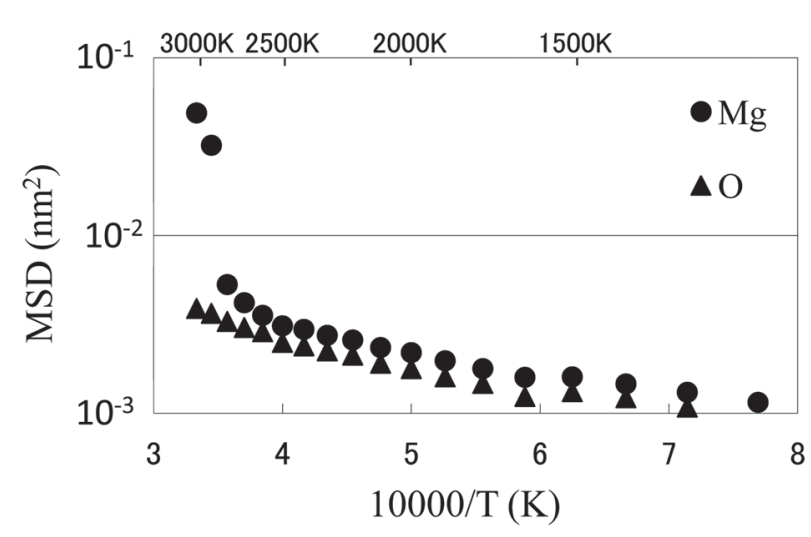

Figure 7. Temperature dependences of MSD at $0 \mathrm{GPa}$.

the duration was not shown in Figure 6. This got into alignment with that the MSD of oxygen ion having constant value.

\subsection{Behvior of Frenkel defect and MSD variations with temperature}

Figures 7 and 8 show temperature dependences of MSD and the appearance frequency of Frenkel defect for magnesium ion at $0 \mathrm{GPa}$, respectively. From 1200 to $2500 \mathrm{~K}$, the MSD of magnesium ion gradually increases with temperature. Around $2600 \mathrm{~K}$, however, the value increases precipitously and jumps to higher value around $2900 \mathrm{~K}$ (Figure 7). Although Frenkel defects cannot be detected in the present study below $2500 \mathrm{~K}$, the defects appear above $2600 \mathrm{~K}$. The frequency increases exponentially with temperature and jumps to higher value around

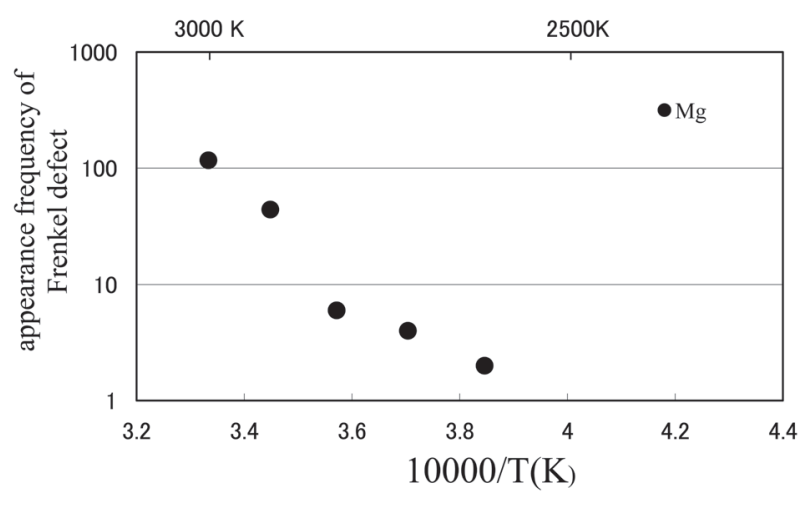

Figure 8. Temperature dependences of the appearance frequency of Frenkel defect for $\mathrm{Mg}$ ion at $0 \mathrm{GPa}$.

$2900 \mathrm{~K}$ (Figure 8). On the other hand, MSD of oxygen ion gradually increases with temperature from 1200 to $3000 \mathrm{~K}$ and Frenkel defects cannot be observed in the present study within the simulated temperature range. Because ionic size of oxygen ion is larger than that of magnesium ion, the formation energy of Frenkel defect and interstitial atom pair for oxygen ion is supposed to be higher than that for magnesium ion. These results show that the MSD value has a strong correlation with the number of Frenkel defects and interstitial atoms. Because Frenkel defects and interstitial atoms interact strongly the diffusion process, the increase of MSD values means the increase of the diffusion coefficient and the formation, the migration and the disappearance of Frenkel defect and interstitial atom cannot be ignored for the diffusion process at high temperature. 


\subsection{Self-diffusion at atmospheric pressure}

Self-diffusion coefficient for magnesium ion was estimated from MSD results during the whole simulated time, although the MSD of magnesium ion had the stepwise change (Figure 6). The estimated values, about $3.5 \times 10^{-13} \mathrm{~m}^{2} / \mathrm{s}$, at $2700 \mathrm{~K}$ are smaller than those calculated by the experimental studies, $3.0 \times 10^{-13} \mathrm{~m}^{2} / \mathrm{s}$ (Wuensch et al. [12]) and $2.6 \times 10^{-12} \mathrm{~m}^{2} / \mathrm{s}$ (Harding and Price [13]), and the other MD simulation study, $8.5 \times 10^{-12} \mathrm{~m}^{2} / \mathrm{s}$ (Ito and Toriumi [3]). At $3000 \mathrm{~K}$, moreover, $4.1 \times 10^{-12} \mathrm{~m}^{2} / \mathrm{s}$ obtained in this study is much smaller than $2.1 \times 10^{-11} \mathrm{~m}^{2} / \mathrm{s}$ estimated by Ito and Toriumi [3]. Because in these experimental studies and the past MD study, the structure included the Schottky defects as initial state, the self-diffusion processes were easy to occur. In the present system, in contrast, the generation process of vacancies which needs the large activation energy are necessary, self-diffusion coefficient estimated from MSD has a small value. Although the self-diffusion coefficient for oxygen ion could be estimated at $2.5 \times 10^{-13} \mathrm{~m}^{2} / \mathrm{s}$ from MSD, on the other hand, the diffusion process could not be detected and it could not be compared with past experiments.

From the monotonous increasing period in Figure 6, e.g., from about 19,000 to about 20,000 MD steps, the local selfdiffusion coefficient for magnesium ion could be estimated roughly at about $4 \times 10^{-10} \mathrm{~m}^{2} / \mathrm{s}$ at $3000 \mathrm{~K}$. This period shows that Frenkel defects and interstitial atoms exited and migrated. This estimated value was larger than that calculated by Ito and Toriumi [3], $2.1 \times 10^{-11} \mathrm{~m}^{2} / \mathrm{s}$. This suggested that diffusion process readily occurred because both Frenkel defects and interstitial atoms exited in the crystal.
We also thank N. Shimobayashi and S. Ohi for helpful comments. This work was partially supported by a Grant-in-Aid for Scientific Research from the Ministry of Education, Science, Sports, and Culture in Japan.

\section{References}

[1] A. James, Geophys. Res. Lett., 30, 1056 (2003). [CrossRef]

[2] A. Lidunka Vočadlo, Phys. Earth Planet. Inter, 88, 193 (1995). [CrossRef]

[3] Yousuke Ito and Mitsuhiro Toriumi, J. Geophys. Res. Solid Earth, 112, B4, B04206 (2007).

[4] A. Miyake, Mineral. J., 20, 189 (1998). [CrossRef]

[5] A. Miyake, N. Shimobayashi, M. Kitamura, Am. Mineral., 89, 1667 (2004).

[6] A. Miyake, J. Am. Ceram. Soc., 88, 121 (2005). [CrossRef]

[7] Katsuyuki Kawamura, "MXDORTO" Japan Chemistry Program Exchange Newsletter, 7(4), 71, (in Japanese) (1996-)

[8] Ralph Walter Graystone Wyckoff, Crystal Structures. Vol. 1. (pp. 87). R.E. Krieger Pub Co. (1982)

[9] A. Miyake, H. Hasegawa, K. Kawamura, M. Kitamura, Acta Crystallogr. A, 54, 330 (1998). [CrossRef]

[10] L. Dubrovinsky, S. Saxena, Phys. Chem. Miner, 24, 547 (1997). [CrossRef]

[11] A. Dewaele, G. Fiquet, D. Andrault, D. Hausermann, $J$. Geophys. Res., 105, B2, 2869 (2000). [CrossRef]

[12] B. J. Wuensch, W. C. Steele, T. Vasilos, J. Chem. Phys., 58, 5258 (1973). [CrossRef]

[13] B. C. Harding, D. M. Price, Philos. Mag., 26, 253 (1972). [CrossRef] 